\title{
Computerized generation of surfaces with optimal approximation to ideal surfaces
}

\author{
F.L. Litvin and N.X. Chen \\ The University of Illinois at Chicago, IL, USA \\ Y. Zhang \\ The University of Michigan at Dearborn, $M I, U S A$ \\ T.J. Krenzer \\ The Gleason Works, Rochester, NY, USA \\ R.F. Handschuh \\ The NASA Lewis Research Center, Cleveland, OH, USA \\ Received 19 March 1992 \\ Revised manuscript received 14 November 1992
}

\begin{abstract}
The authors propose a new method for generation (by grinding or cutting) of a surface $\left(\Sigma_{g}\right)$ with the optimal approximation to the theoretical (ideal) surface $\left(\Sigma_{p}\right)$. The method is based on the following ideas: (1) A region of space is swept out by the tool surface $\Sigma_{t}$ performing certain motions with respect to $\Sigma_{p}$. The surface of the tool (as grinding wheel or cutter) is a surface of revolution with a circular arc in axial section, and a circular cone in particular cases. (2) The space swept out by $\Sigma_{t}$ is considered as a family of surfaces $\Sigma_{t}$, and the envelope to this family is surface $\Sigma_{g}$ (generated surface) that must be in optimal approximation to the theoretical surface $\Sigma_{p}$. (3) The continuous varied setting and orientation of $\Sigma_{1}$ with respect to $\Sigma_{p}$ are executed by a multi-degree-of-freedom machine, that is a computer numerical controlled (CNC) machine. The approach developed can be applied for grinding of face-gears, helical involute gears with modified topology, ruled undeveloped surfaces and others. An example of application is considered.
\end{abstract}

\section{Introduction}

The development of multi-degree-of-freedom machines, numerically controlled by computer (CNC) machines, has opened new perspectives for the generation of surfaces with new topology, and the generation of a surface $\left(\Sigma_{\mathrm{g}}\right)$ that must be optimal approximation to the theoretical (ideal) surface $\left(\Sigma_{\mathrm{p}}\right)$.

The authors propose a method for generation of $\Sigma_{\mathrm{g}}$ (with optimal approximation to $\Sigma_{\mathrm{p}}$ ) based on the following ideas:

(1) A mean line $L_{\mathrm{m}}$ on the ideal surface $\Sigma_{\mathrm{p}}$ is chosen as shown in Fig. 1.

(2) The tool surface $\Sigma_{t}$ is a properly designed surface of revolution (in particular cases $\Sigma_{t}$ is a circular cone as shown in Fig. 1) that moves along $L_{\mathrm{m}}$. Surfaces $\Sigma_{\mathrm{t}}$ and $\Sigma_{\mathrm{p}}$ are in continuous tangency along $L_{\mathrm{m}} ; M$ is the current point of tangency (Fig. 1). The orientation of $\Sigma_{\mathrm{t}}$ with respect to $\Sigma_{\mathrm{p}}$ (determined with angle $\beta$ ) is continuously varying. Angle $\beta$ at the current point $M$ of tangency is formed by the tangents $t_{\mathrm{f}}$ and $t_{\mathrm{b}}$ to $L_{\mathrm{m}}$ and the tool generatrix, respectively (Fig. 1). Tangents $t_{\mathrm{f}}$ and $t_{\mathrm{b}}$ form a plane $\Pi$ that is tangent to $\Sigma_{\mathrm{t}}$ and $\Sigma_{\mathrm{p}}$ at point $M$. 


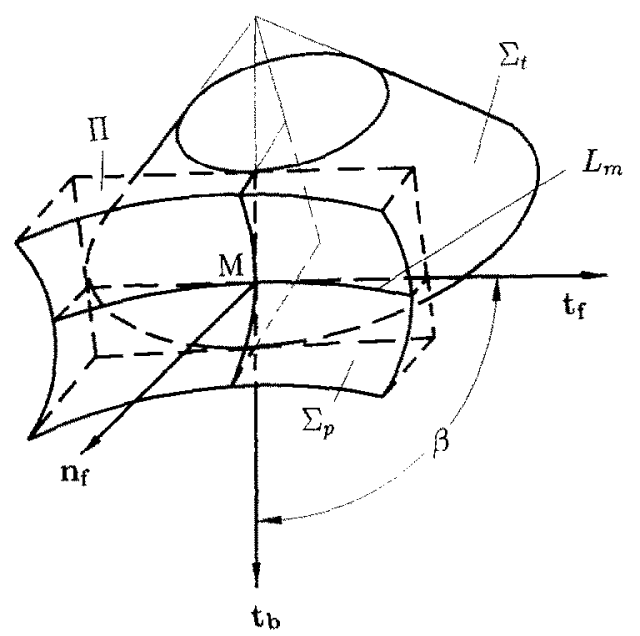

Fig. 1. Installment and orientation of tool surface $\Sigma_{\mathrm{t}}$ with respect to ideal surface $\Sigma_{\mathrm{p}}$.

(3) The tool surface $\Sigma_{t}$ in its motion with respect to $\Sigma_{p}$ swept out a region of space as a family of surfaces $\Sigma_{\mathrm{t}}$. The envelope to the family of $\Sigma_{\mathrm{t}}$ is the surface $\Sigma_{\mathrm{g}}$ (the ground, cut surface) that is in tangency with the theoretical surface $\Sigma_{\mathrm{p}}$ at any point $M$ of $L_{\mathrm{m}}$ and must be in optimal approximation to $\Sigma_{\mathrm{p}}$ in any direction that differs from $L_{\mathrm{m}}$.

(4) The optimal approximation of $\Sigma_{\mathrm{g}}$ to $\Sigma_{\mathrm{p}}$ is obtained by variation of angle $\beta$ (Fig. 1).

(5) The continuous tangency of tool surface $\Sigma_{\mathrm{t}}$ with $\Sigma_{\mathrm{p}}$ and properly varied orientation of $\Sigma_{\mathrm{t}}$ can be obtained by the execution of required motions of the tool by a computer controlled multi-degreeof-freedom machine. One of these degrees of freedom, rotation of the tool about its axis, provides the desired velocity of grinding (cutting) and is not related to the process for generation of $\Sigma_{g}$.

The paper covers the following topics:

(1) Determination of the equation of meshing between the tool surface $\Sigma_{t}$ and the generated surface $\Sigma_{\mathrm{g}}$. The term 'equation of meshing' is used in the theory of gearing [1] and is represented as $f\left(u_{\mathrm{t}}, \theta_{\mathrm{t}}, \theta_{\mathrm{p}}\right)=0$ where $\left(u_{\mathrm{t}}, \theta_{\mathrm{t}}\right)$ are the Gaussian coordinates of $\Sigma_{\mathrm{t}}$ and $\theta_{\mathrm{p}}$ is the generalized parameter of motion. The equation of meshing provides the neccssary condition of existence of the envelope to the family of surfaces.

(2) Determination of the generated surface $\Sigma_{\mathrm{g}}$ as the envelope to the family of surfaces $\Sigma_{\mathrm{t}}$ swept out by the tool. Surface $\Sigma_{\mathrm{g}}$ coincides with the theoretical (ideal) surface $\Sigma_{\mathrm{p}}$ along the mean line $L_{\mathrm{m}}$ and deviates from $\Sigma_{p}$ out of $L_{m}$.

(3) Determination of deviations of $\Sigma_{\mathrm{g}}$ from $\Sigma_{\mathrm{p}}$ (in regions that differ from $L_{\mathrm{m}}$ ) and minimizations of $\Sigma_{\mathrm{g}}$ deviations for optimal approximation of $\Sigma_{\mathrm{g}}$ to $\Sigma_{\mathrm{p}}$.

(4) Determination of curvatures of $\Sigma_{\mathrm{g}}$ that are required when the simulation of meshing and contact of two mating surfaces are considered.

(5) Execution of required motions of $\Sigma_{\mathrm{t}}$ with respect to $\Sigma_{\mathrm{p}}$ by application of a multi-degree-freedom, computer numerically controlled machine.

The authors have developed an effective approach for the derivation of the necessary condition for the existence of the envelope $\Sigma_{g}$ using the idea of motion of the Darboux-Frenet trihedron along $L_{m}$, the chosen mean line of $\Sigma_{p}$.

An additional effective approach has been proposed and developed for determination of curvatures of generated surface $\vec{\Sigma}_{g}$. This approach is based on the fact that the normal curvatures and surface torsions (geodesic torsions) of $\Sigma_{\mathrm{g}}$ are: (i) equal to the normal curvatures and surface torsions of $\Sigma_{\mathrm{p}}$ along $L_{\mathrm{m}}$; and (ii) equal to the normal curvatures and surface torsions of tool surface $\Sigma_{\mathrm{t}}$ along the characteristic $L_{\mathrm{g}}$ (the instantaneous line of tangency of $\Sigma_{\mathrm{t}}$ and $\Sigma_{\mathrm{g}}$ ). 


\section{Mean line on the ideal surface $\Sigma_{\mathrm{p}}$}

The ideal surface $\Sigma_{\mathrm{p}}$ is considered as a regular one and is represented as

$$
r_{\mathrm{p}}\left(u_{\mathrm{p}}, \theta_{\mathrm{p}}\right) \in C^{2}, \quad \frac{\partial r_{\mathrm{p}}}{\partial u_{\mathrm{p}}} \times \frac{\partial r_{\mathrm{p}}}{\partial \theta_{\mathrm{p}}} \neq 0, \quad\left(u_{p}, \theta_{p}\right) \in E,
$$

where $\left(u_{p}, \theta_{p}\right)$ are the Gaussian coordinates of $\Sigma_{p}$.

The unit normal to $\Sigma_{\mathrm{p}}$ is represented as

$$
n_{\mathrm{p}}=\frac{\boldsymbol{N}_{\mathrm{p}}}{\left|\boldsymbol{N}_{\mathrm{p}}\right|}, \quad \boldsymbol{N}_{\mathrm{p}}=\frac{\partial \boldsymbol{r}_{\mathrm{p}}}{\partial u_{\mathrm{p}}} \times \frac{\partial \boldsymbol{r}_{\mathrm{p}}}{\partial \theta_{\mathrm{p}}} .
$$

The determination of the mean line $L_{\mathrm{m}}$ is based on the following procedure:

(i) Initially, we determine numerically $n$ points on the surface $\Sigma_{p}$ that will belong approximately to the desired mean line $L_{\mathrm{m}}$.

(ii) Then, we can derive a polynomial function

$$
u_{\mathrm{p}}\left(\theta_{\mathrm{p}}\right)=\sum_{j=1}^{n} a_{j} \theta_{\mathrm{p}}^{(n-j)}
$$

that will relate the surface parameters $\left(u_{\mathrm{p}}, \boldsymbol{\theta}_{\mathrm{p}}\right)$ for the $n$ points of the mean line on $\Sigma_{\mathrm{p}}$.

The mean line $L_{\mathrm{m}}$, tangent $\boldsymbol{T}_{\mathrm{p}}$ and unit tangent $t_{\mathrm{p}}$ to the mean line are represented as follows:

$$
\boldsymbol{r}_{\mathrm{p}}\left(u_{\mathrm{p}}\left(\theta_{\mathrm{p}}\right), \theta_{\mathrm{p}}\right), \quad \boldsymbol{T}_{\mathrm{p}}=\frac{\partial \boldsymbol{r}_{\mathrm{p}}}{\partial \theta_{\mathrm{p}}}+\frac{\partial \boldsymbol{r}_{\mathrm{p}}}{\partial u_{\mathrm{p}}} \frac{\mathrm{d} u_{\mathrm{p}}}{\mathrm{d} \theta_{\mathrm{p}}}, \quad \boldsymbol{t}_{\mathrm{p}}=\frac{\boldsymbol{T}_{\mathrm{p}}}{\left|\boldsymbol{T}_{\mathrm{p}}\right|} .
$$

The constraint for $t_{\mathrm{p}}$ is that it must be of the same sign and differ from zero at the same intervals of interpolation.

\section{Tool surface}

The tool surface $\Sigma_{t}$ is represented in coordinate system $S_{\mathrm{t}}$ rigidly connected to the tool by the following equations:

$$
x_{\mathrm{t}}=x_{\mathrm{t}}\left(u_{\mathrm{t}}\right) \cos \theta_{\mathrm{t}}, \quad y_{\mathrm{t}}=x_{\mathrm{t}}\left(u_{\mathrm{t}}\right) \sin \theta_{\mathrm{t}}, \quad z_{\mathrm{t}}=z_{\mathrm{t}}\left(u_{\mathrm{t}}\right),
$$

The axial section of $\Sigma_{\mathrm{t}}$ obtained by taking $\theta_{\mathrm{t}}=0$ represents a circular arc, or a straight line in the case where $\Sigma_{t}$ is a circular cone.

The surface unit normal is determined as

$$
\boldsymbol{n}_{\mathrm{t}}=\frac{N_{\mathrm{t}}}{\left|N_{\mathrm{t}}\right|}, \quad N_{\mathrm{t}}=\frac{\partial \boldsymbol{r}_{\mathrm{t}}}{\partial \theta_{\mathrm{t}}} \times \frac{\partial \boldsymbol{r}_{\mathrm{t}}}{\partial u_{\mathrm{t}}}
$$

\section{Equation of meshing between $\Sigma_{\mathrm{t}}$ and $\Sigma_{\mathrm{g}}$}

Equation of meshing represents the necessary condition of existence of envelope $\Sigma_{\mathrm{g}}$ to the family of surfaces $\Sigma_{t}$ that is swept out by the tool surface $\Sigma_{t}$.

In the theory of gearing [1], the equation of meshing can be derived by using the equation 


$$
N_{i}^{(\mathrm{t})} \cdot \boldsymbol{v}_{i}^{(\mathrm{tg})}=0
$$

Here, $i$ indicates the coordinate system where the vectors of the scalar product are represented, $N^{(1)}$ is the normal to surface $\Sigma_{t}$; and $v^{(\mathrm{tg})}$ is the relative velocity in the motion of $\Sigma_{\mathrm{t}}$ with respect to $\Sigma_{\mathrm{g}}$.

Henceforth, we consider two basic coordinate systems, $S_{\mathrm{t}}$ and $S_{\mathrm{p}}$, that are rigidly connected to the tool surface $\Sigma_{\mathrm{t}}$ and the ideal surface $\Sigma_{\mathrm{p}}$. In addition to $\Sigma_{\mathrm{t}}$, we consider two trihedrons: $S_{\mathrm{b}}\left(t_{\mathrm{b}}, \boldsymbol{d}_{\mathrm{b}}, \boldsymbol{n}_{\mathrm{b}}\right)$ and $S_{\mathrm{f}}\left(\boldsymbol{t}_{\mathrm{f}}, \boldsymbol{d}_{\mathrm{f}}, \boldsymbol{n}_{\mathrm{f}}\right)$. Trihedron $S_{\mathrm{b}}$ is rigidly connected to $\Sigma_{\mathrm{f}}$ and coordinate system $S_{\mathrm{t}}$ (Fig. 2). Here, $O_{\mathrm{b}}$ is the point of the chosen generatrix of $\Sigma_{\mathrm{t}}$ where the trihedron is located, $t_{\mathrm{b}}$ is the tangent to the generatrix at $O_{\mathrm{b}} ; \boldsymbol{n}_{\mathrm{b}}$ is the surface unit normal of $\Sigma_{\mathrm{t}}$ at $O_{\mathrm{b}}, \boldsymbol{d}_{\mathrm{b}}=\boldsymbol{n}_{\mathrm{b}} \times \boldsymbol{t}_{\mathrm{b}}$, and vectors $\boldsymbol{t}_{\mathrm{b}}$ and $\boldsymbol{d}_{\mathrm{b}}$ form the tangent plane to $\Sigma_{\mathrm{t}}$ at $O_{\mathrm{b}}$. Trihedron $S_{\mathrm{f}}$ moves along the mean line $L_{\mathrm{m}}$ (Fig. 3); $t_{\mathrm{f}}$ is the tangent to the mean line $L_{\mathrm{m}}$ at current point $M$ (Fig. 3 ); $n_{\mathrm{f}}$ is the surface unit normal to $\Sigma_{\mathrm{p}}$ at point $M ; \boldsymbol{d}_{\mathrm{f}}=\boldsymbol{n}_{\mathrm{f}} \times \boldsymbol{t}_{\mathrm{f}}$; vectors $\boldsymbol{t}_{\mathrm{f}}$ and $\boldsymbol{d}_{\mathrm{f}}$ form the tangent plane to $\Sigma_{\mathrm{p}}$ at point $M$.

The tool with surface $\Sigma_{\mathrm{t}}$ and trihedron $S_{\mathrm{t}}$ moves along mean line $L_{\mathrm{m}}$ of $\Sigma_{\mathrm{p}}$ and $O_{\mathrm{b}}$ coincides with current point $M$ of mean line $L_{\mathrm{m}}$. Surfaces $\Sigma_{\mathrm{t}}$ and $\Sigma_{\mathrm{p}}$ are in tangency at any current point $M$ of mean line $L_{\mathrm{m}}$. The orientation of $S_{\mathrm{b}}$ with respect to $S_{\mathrm{f}}$ is determined with angle $\beta$ that is varied in the process for generation.

We start the derivations with the case where $\Sigma_{\mathrm{t}}$ is a circular cone (Fig. 4 ). The angular velocity $\omega_{\mathrm{f}}$ of rotation of $S_{\mathrm{f}}$ with respect to $S_{\mathrm{p}}$ is represented as $[2,3]$ :

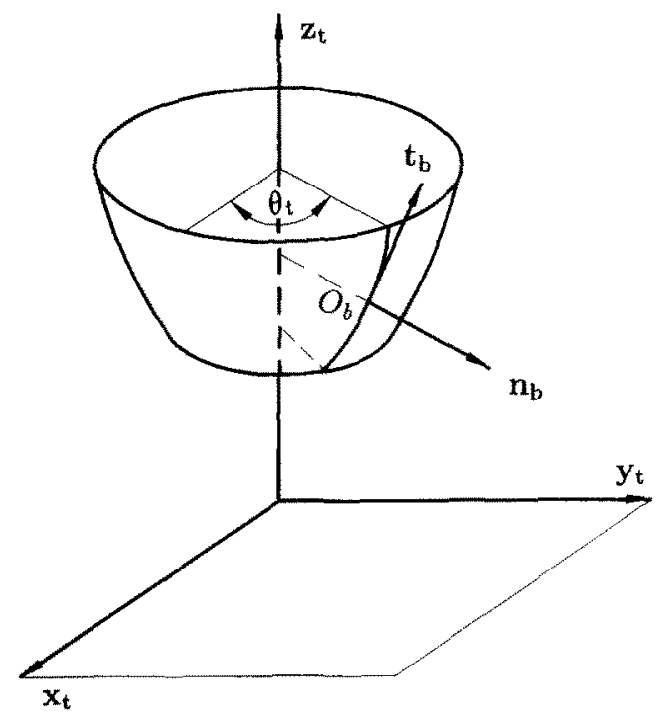

Fig. 2. Tool surface $\Sigma_{i}$.

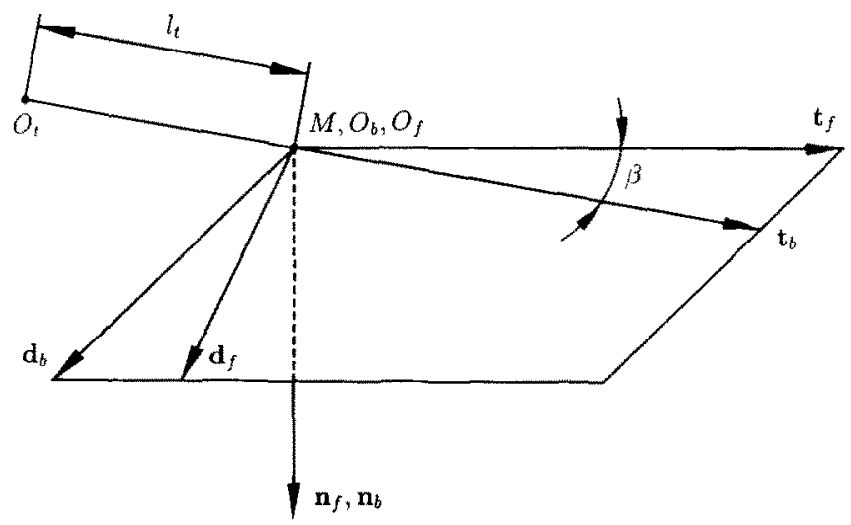

Fig. 3. Orientation of trihedron $S_{\mathrm{b}}$ with respect to $S_{\mathrm{f}}$. 


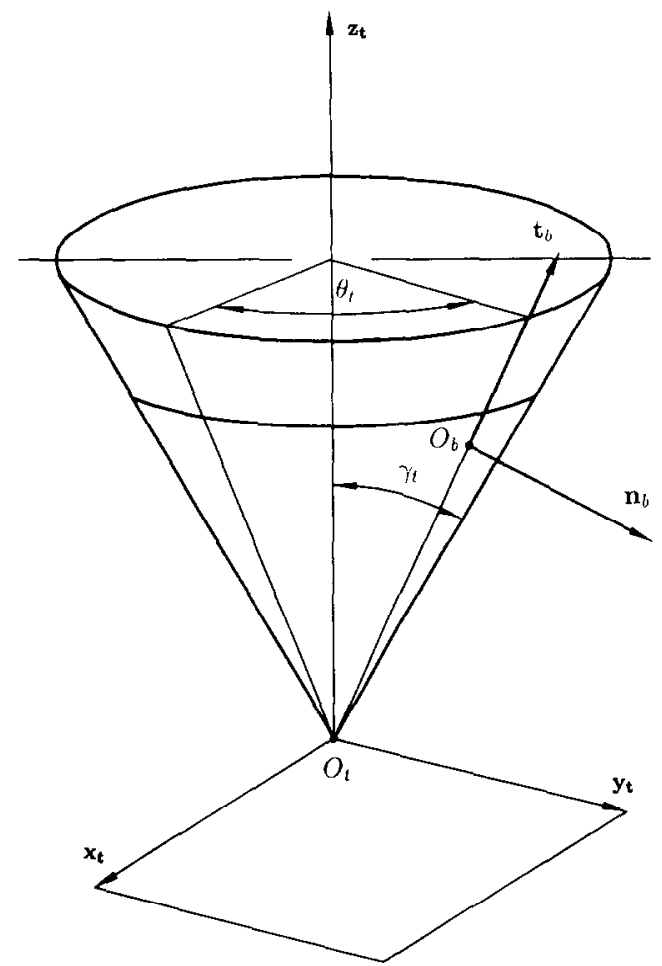

Fig. 4. Surface of grinding tool cone.

$$
\omega_{\mathrm{f}}=\left(t t_{\mathrm{f}}-k_{n} d_{\mathrm{f}}+k_{g} n_{\mathrm{f}}\right) \frac{\mathrm{d} s}{\mathrm{~d} t} .
$$

Here, $t$ is the surface torsion (geodesic torsion), $k_{\mathrm{n}}$ and $k_{\mathrm{g}}$ are the normal and geodesic curvatures of surface $\Sigma_{\mathrm{p}}$ at the current point $M$ of the mean line $L_{\mathrm{m}}$, ds is the infinitesimal displacement along $L_{\mathrm{m}}$. The definition of surface torsion is given in [3]; the concept of the equivalent term 'geodesic torsion' is also discussed in [2].

The angular velocity $\boldsymbol{\Omega}_{\mathrm{f}}$ of trihedron $S_{\mathrm{b}}$ is represented in $S_{\mathrm{f}}$ as

$$
\boldsymbol{\Omega}_{\mathrm{f}}=\omega_{\mathrm{f}}+\frac{\mathrm{d} \beta}{\mathrm{d} t} n_{\mathrm{f}}=\left[\begin{array}{lll}
t & -k_{\mathrm{n}} & k_{\mathrm{g}}+\frac{\mathrm{d} \beta}{\mathrm{d} s}
\end{array}\right]^{\mathrm{t}} \frac{\mathrm{d} s}{\mathrm{~d} t} .
$$

The orientation of cone $\Sigma_{t}$ is determined by function $\beta\left(\theta_{p}\right)$ and

$$
\frac{\mathrm{d} \beta}{\mathrm{d} s}=\frac{\mathrm{d} \beta}{\mathrm{d} \theta_{\mathrm{p}}} \frac{\mathrm{d} \theta_{\mathrm{p}}}{\mathrm{d} s}=\left(\frac{\mathrm{d} \beta}{\mathrm{d} \theta_{\mathrm{p}}}\right) \frac{1}{\left|\boldsymbol{T}_{\mathrm{p}}\right|},
$$

where $T_{\mathrm{p}}$ is the tangent to the mean line $L_{\mathrm{m}}$ at current point $M$.

The transformations of vector components in transition from $S_{\mathrm{t}}$ to $S_{\mathrm{b}}$ and $S_{\mathrm{f}}$ are represented by $3 \times 3$ matrix operators $\boldsymbol{L}_{\mathrm{bt}}$ and $\boldsymbol{L}_{\mathrm{fb}}$. Here,

$$
\boldsymbol{L}_{\mathrm{fb}}=\left[\begin{array}{ccc}
\cos \beta & -\sin \beta & 0 \\
\sin \beta & \cos \beta & 0 \\
0 & 0 & 1
\end{array}\right]
$$




$$
\boldsymbol{L}_{\mathrm{bt}}=\left[\begin{array}{ccc}
\sin \gamma_{\mathrm{t}} \cos \theta_{\mathrm{t}} & \sin \gamma_{\mathrm{t}} \sin \theta_{\mathrm{t}} & \cos \gamma_{\mathrm{t}} \\
\sin \theta_{\mathrm{t}} & -\cos \theta_{\mathrm{t}} & 0 \\
\cos \gamma_{\mathrm{t}} \cos \theta_{\mathrm{t}} & \cos \gamma_{\mathrm{t}} \sin \theta_{\mathrm{t}} & -\sin \gamma_{\mathrm{t}}
\end{array}\right] .
$$

The cone surface $\Sigma_{\mathrm{t}}$ is represented in $S_{\mathrm{t}}$ as follows (Fig. 4):

$$
r_{\mathrm{t}}=u_{\mathrm{t}}\left[\begin{array}{lll}
\sin \gamma_{\mathrm{t}} \cos \theta_{\mathrm{t}} & \sin \gamma_{\mathrm{t}} \sin \theta_{\mathrm{t}} & \cos \gamma_{\mathrm{t}}
\end{array}\right]^{\mathrm{t}}
$$

where $\left(u_{t}, \theta_{t}\right)$ are the surface parameters and $\gamma_{t}$ is the cone apex angle.

The unit normal to the cone surface is

$$
\boldsymbol{n}_{\mathrm{t}}=u_{\mathrm{t}}\left[\begin{array}{ccc}
\cos \gamma_{\mathrm{t}} \cos \theta_{\mathrm{t}} & \cos \gamma_{\mathrm{t}} \sin \theta_{\mathrm{t}} & -\sin \gamma_{\mathrm{t}}
\end{array}\right]^{\mathrm{t}}
$$

The required equation of meshing (necessary condition of existence of envelope $\Sigma_{\mathrm{g}}$ ) is represented in the form

where

$$
\boldsymbol{n}_{\mathrm{f}}^{(\mathrm{t})} \cdot \boldsymbol{v}_{\mathrm{f}}^{(\mathrm{tg})}=0
$$

$$
n_{\mathrm{f}}^{(\mathrm{t})}=L_{\mathrm{ft}} n_{\mathrm{t}} \text {. }
$$

The derivation of the expression $\boldsymbol{v}_{\mathrm{f}}^{(\mathrm{tg})}$ is simplified while taking into account the following considerations:

(a) The relative velocity vector $v_{\mathrm{f}}^{(\mathrm{tg})}$ can be represented as

$$
\boldsymbol{v}_{\mathrm{f}}^{(\mathrm{tg})}=\boldsymbol{\Omega}_{\mathrm{f}}^{(\mathrm{s})} \boldsymbol{r}_{\mathrm{f}}^{(\mathrm{t})}+\frac{\mathrm{d} s}{\mathrm{~d} t} \boldsymbol{t}_{\mathrm{f}}
$$

Here, $\boldsymbol{\Omega}_{\mathrm{f}}^{(\mathrm{s})}$ is the skew-symmetric matrix represented as

$$
\boldsymbol{\Omega}_{\mathrm{f}}^{(\mathrm{s})}=\left[\begin{array}{ccc}
0 & -\omega_{3} & \omega_{2} \\
\omega_{3} & 0 & -\omega_{1} \\
-\omega_{2} & \omega_{1} & 0
\end{array}\right]
$$

Vector $\boldsymbol{\Omega}_{\mathrm{f}}$ is represented by

$$
\boldsymbol{\Omega}_{\mathrm{f}}=\omega_{1} t_{\mathrm{t}}+\omega_{2} d_{\mathrm{f}}+\omega_{3} \boldsymbol{n}_{\mathrm{f}}=\left[\begin{array}{lll}
t & -k_{\mathrm{n}} & k_{\mathrm{g}}+\frac{\mathrm{d} \beta}{\mathrm{d} s}
\end{array}\right]^{\mathrm{t}} \frac{\mathrm{d} s}{\mathrm{~d} t} .
$$

(b) Consider that point $N$ on surface $\Sigma_{\mathrm{t}}$ is the point of the characteristic (the line of tangency of $\Sigma_{\mathrm{t}}$ and the generated surface $\Sigma_{\mathrm{g}}$ ). Certainly, the equation of meshing must be satisfied for point $N$.

The position vector $\overline{O_{\mathrm{f}} N}$ can be represented as

$$
\overline{O_{\mathrm{f}} N}=\overline{O_{\mathrm{t}} N}-\overline{O_{\mathrm{t}} O_{\mathrm{f}}},
$$

Here, $\overline{O_{\mathrm{t}} N}$ is the position vector of point $N$ that is drawn from the origin $O_{\mathrm{t}}$ of $S_{\mathrm{t}}$ to $N$; vector $\overline{O_{\mathrm{t}} N}$ is represented as

where

$$
\overline{O_{\mathrm{t}} N}=u_{\mathrm{t}} e_{\mathrm{t}}=u_{\mathrm{t}}\left(\sin \gamma_{\mathrm{t}} \cos \theta_{\mathrm{t}} i_{\mathrm{t}}+\sin \gamma_{\mathrm{t}} \sin \theta_{\mathrm{t}} j_{\mathrm{t}}+\cos \gamma_{\mathrm{t}} k_{\mathrm{t}}\right),
$$

$$
\boldsymbol{e}_{\mathrm{t}}=\frac{\frac{\partial}{\partial u_{\mathrm{t}}}\left(\boldsymbol{r}_{\mathrm{t}}\right)}{\left|\frac{\partial}{\partial u_{\mathrm{t}}}\left(\boldsymbol{r}_{\mathrm{t}}\right)\right|}
$$


is the unit vector of cone generatrix $\overline{O_{1} N}$.

Vector $\overline{O_{\mathrm{t}} O_{\mathrm{f}}}$ (Fig. 4) is represented in $S_{\mathrm{b}}$ as

$$
\overline{O_{\mathrm{t}} O_{\mathrm{f}}}=l_{\mathrm{t}} i_{\mathrm{b}}
$$

where $l_{\mathrm{t}}=\left|\overline{O_{\mathrm{t}} O_{\mathrm{f}}}\right|$.

Vector $\overline{O_{\mathrm{f}} N}$ is represented in $S_{\mathrm{f}}$ using the matrix equation

$$
r_{\mathrm{f}}^{(\mathrm{t})}=u_{\mathrm{t}} L_{\mathrm{ft}} e_{\mathrm{t}}-l_{\mathrm{t}} L_{\mathrm{fb}} i_{\mathrm{b}}
$$

(c) We now represent the equation of meshing as

$$
n_{\mathrm{f}}^{(\mathrm{t})} \cdot \boldsymbol{v}_{\mathrm{f}}^{(\mathrm{ct})}=\left\{\boldsymbol{n}_{\mathrm{f}}^{(\mathrm{t})} \cdot\left[\boldsymbol{\Omega}^{(\mathrm{s})}\left(u_{\mathrm{t}} L_{\mathrm{ft}} \boldsymbol{e}_{\mathrm{t}}-l_{\mathrm{t}} \boldsymbol{L}_{\mathrm{fb}} i_{\mathrm{b}}\right)\right]+\boldsymbol{n}_{\mathrm{f}}^{(\mathrm{t})} \cdot \boldsymbol{t}_{\mathrm{f}}\right\} \frac{\mathrm{d} s}{\mathrm{~d} t}=0
$$

(d) Further simplification of the equation of meshing is based on the following rule for operations with skew-symmetric matrices [4]:

$$
A^{\mathrm{t}} B^{(\mathrm{s})} A=C^{(\mathrm{s})}
$$

where $\boldsymbol{B}^{(\mathrm{s})}$ and $\boldsymbol{C}^{(\mathrm{s})}$ designate skew-symmetric matrices, $\boldsymbol{A}^{\mathrm{t}}$ is the transpose matrix for $\boldsymbol{A}$.

Considering that elements of $\boldsymbol{B}^{(\mathrm{s})}$ are represented in terms of components of the vector

$$
\boldsymbol{b}=\left[\begin{array}{lll}
b_{1} & b_{2} & b_{3}
\end{array}\right]^{\mathbf{t}},
$$

we obtain that the elements of skew-symmetric matrix $C^{(\mathrm{s})}$ are represented in terms of the components of vector $c$, where

$$
\left[\begin{array}{lll}
c_{1} & c_{2} & c_{3}
\end{array}\right]^{\mathrm{t}}=-\boldsymbol{A}^{\mathrm{t}}\left[\begin{array}{lll}
b_{1} & b_{2} & b_{3}
\end{array}\right]^{\mathrm{t}} .
$$

Using the above considerations and eliminating $\mathrm{d} s / \mathrm{d} t$, the final expression of the equation of meshing can be represented as

where

$$
\boldsymbol{n}_{\mathrm{f}}^{(\mathrm{t})} \cdot \boldsymbol{v}_{\mathrm{f}}^{(\mathrm{tg})}=f\left(u_{\mathrm{t}}, \theta_{\mathrm{t}}, \theta_{\mathrm{p}}\right)=u_{\mathrm{t}} \boldsymbol{n}_{\mathrm{t}}^{\mathrm{t}} A^{(\mathrm{s})} e_{\mathrm{t}}-l_{\mathrm{t}} \boldsymbol{n}_{\mathrm{t}}^{\mathrm{t}} B^{(\mathrm{s})} i_{\mathrm{b}}+\boldsymbol{n}_{\mathrm{t}}^{\mathrm{t}} \boldsymbol{L}_{\mathrm{ft}}^{\mathrm{t}} \boldsymbol{t}_{\mathrm{f}}=0
$$

$$
\begin{aligned}
& \boldsymbol{A}^{(\mathrm{s})}=\boldsymbol{L}_{\mathrm{ft}}^{\mathrm{t}} \boldsymbol{\Omega}_{\mathrm{f}}^{(\mathrm{s})} \boldsymbol{L}_{\mathrm{ft}}, \quad \boldsymbol{B}^{(\mathrm{s})}=\boldsymbol{L}_{\mathrm{fb}}^{\mathrm{t}} \boldsymbol{\Omega}_{\mathrm{f}}^{(\mathrm{s})} \boldsymbol{L}_{\mathrm{fb}}, \\
& \boldsymbol{A}^{(\mathrm{s})}=\left[\begin{array}{ccc}
0 & -a_{3} & a_{2} \\
a_{3} & 0 & -a_{1} \\
-a_{2} & a_{1} & 0
\end{array}\right], \\
& {\left[\begin{array}{l}
a_{1} \\
a_{2} \\
a_{3}
\end{array}\right]=-\left[\begin{array}{cc}
t \cos \beta \sin \gamma_{\mathrm{t}}-k_{\mathrm{n}} \sin \beta+\left(k_{\mathrm{g}}+\frac{\mathrm{d} \beta}{\mathrm{d} s}\right) \cos \beta \cos \gamma_{\mathrm{t}} \\
t \sin \beta \sin \gamma_{\mathrm{t}}+k_{\mathrm{n}} \cos \beta+\left(k_{\mathrm{g}}+\frac{\mathrm{d} \beta}{\mathrm{d} s}\right) \sin \beta \cos \gamma_{\mathrm{t}}
\end{array}\right],} \\
& \boldsymbol{B}^{(\mathrm{s})}=\left[\begin{array}{ccc}
0 & t \cos \gamma_{\mathrm{t}}-\left(k_{\mathrm{g}}+\frac{\mathrm{d} \beta}{\mathrm{d} s}\right) \sin \gamma_{\mathrm{t}} \\
b_{3} & 0 & -b_{3} \\
-b_{2} & b_{1} & 0
\end{array}\right], \quad\left[\begin{array}{c}
b_{1} \\
b_{2} \\
b_{3}
\end{array}\right]=\left[\begin{array}{c}
-t \cos \beta+k_{\mathrm{n}} \sin \beta \\
t \sin \beta+k_{\mathrm{n}} \cos \beta \\
-\left(k_{\mathrm{g}}+\frac{\mathrm{d} \beta}{\mathrm{d} s}\right)
\end{array}\right] .
\end{aligned}
$$


The family of characteristics $L_{\mathrm{g}}$, the instantaneous lines of tangency of $\Sigma_{\mathrm{t}}$ and $\Sigma_{\mathrm{g}}$, is represented in $S_{\mathrm{t}}$ by the cquations

$$
r_{\mathrm{r}}=r_{\mathrm{t}}\left(u_{\mathrm{t}}, \theta_{\mathrm{t}}\right), \quad f\left(u_{\mathrm{t}}, \theta_{\mathrm{t}}, \theta_{\mathrm{p}}\right)=0,
$$

where $\theta_{\mathrm{p}}$ is the parameter of the family of $L_{\mathrm{g}}$. Taking $\theta_{\mathrm{p}}=\theta_{\mathrm{p}}^{(i)}(i=1,2, \ldots, n)$, we obtain the current characteristics on the surface $\Sigma_{t}$.

It is easy to verify that the equation of meshing between $\Sigma_{\mathrm{t}}$ and $\Sigma_{\mathrm{g}}$ is satisfied for the current point $M$ of the mean line $L_{\mathrm{m}}$ on the ideal surface $\Sigma_{\mathrm{p}}$. This means that the characteristic $L_{\mathrm{g}}$ intersects $L_{\mathrm{m}}$ at point $M$, for which we can take $\theta_{t}=0$ since $\Sigma_{t}$ is a surface of revolution. In the case where $\Sigma_{t}$ is a circular cone (Fig. 4), we can take for point $M$ that $u_{\mathrm{t}}=\left|\overline{O_{\mathrm{t}} O_{\mathrm{b}}}\right|=l_{\mathrm{t}}$.

The approach discussed above for the derivation of equation of meshing can be easily extended for application in the more general case where the tool surface is a general surface of revolution.

\section{Determination of generated surface $\Sigma_{\mathrm{g}}$}

The ground surface $\Sigma_{\mathrm{g}}$ is generated as the envelope to the family of tool surfaces $\Sigma_{\mathrm{t}}$; surface $\Sigma_{\mathrm{g}}$ is represented in $S_{\mathrm{p}}$ by the following equations:

$$
r_{\mathrm{g}}^{(\mathrm{p})}\left(u_{\mathrm{p}}\left(\theta_{\mathrm{p}}\right), \theta_{\mathrm{p}}, u_{\mathrm{t}}, \theta_{\mathrm{t}}\right)=\boldsymbol{L}_{\mathrm{pf}} \boldsymbol{r}_{\mathrm{f}}^{(\mathrm{t})}+\boldsymbol{r}_{\mathrm{p}}^{(\mathrm{M})}\left(u_{\mathrm{p}}\left(\theta_{\mathrm{p}}\right), \theta_{\mathrm{p}}\right), \quad f\left(u_{\mathrm{t}}, \theta_{\mathrm{t}}, \theta_{\mathrm{p}}\right)=0
$$

where $f\left(u_{\mathrm{t}}, \theta_{\mathrm{t}}, \theta_{\mathrm{p}}\right)=0$ is the equation of meshing; $\boldsymbol{r}_{\mathrm{f}}^{(\mathrm{t})}\left(u_{\mathrm{t}}, \theta_{\mathrm{t}}\right)$ is the equation of the tool surface $\Sigma_{\mathrm{t}}$ represented in $S_{\mathrm{f}} ; r_{\mathrm{p}}^{(\mathrm{M})}\left(u_{\mathrm{p}}\left(\theta_{\mathrm{p}}\right), \theta_{\mathrm{p}}\right)$ is the vector function that represents in $S_{\mathrm{p}}$ the mean line $L_{\mathrm{m}} ;$ the $3 \times 3$ matrix operator $\boldsymbol{L}_{\mathrm{pf}}$ which transforms vectors in transition from $S_{\mathrm{f}}$ to $S_{\mathrm{p}}$ is represented as

where

$$
\boldsymbol{L}_{\mathrm{pf}}=\left[\begin{array}{lll}
t_{\mathrm{p} x} & d_{\mathrm{p} x} & n_{\mathrm{p} x} \\
t_{\mathrm{p} y} & d_{\mathrm{p} y} & n_{\mathrm{p} y} \\
t_{\mathrm{p} z} & d_{\mathrm{p} z} & n_{\mathrm{p} z}
\end{array}\right],
$$

$$
t_{\mathrm{p}}=\frac{\frac{\partial}{\partial \theta_{\mathrm{p}}}\left(r_{\mathrm{p}}^{(\mathrm{M})}\right)}{\left|\frac{\partial}{\partial \theta_{\mathrm{p}}}\left(r_{\mathrm{p}}^{(\mathrm{M})}\right)\right|}
$$

is the unit target to the mean line $L_{\mathrm{m}}$;

$$
\begin{aligned}
& n_{\mathrm{p}}= \pm \frac{\frac{\partial \boldsymbol{r}_{\mathrm{p}}}{\partial u_{\mathrm{p}}} \times \frac{\partial \boldsymbol{r}_{\mathrm{p}}}{\partial \theta_{\mathrm{p}}}}{\left|\frac{\partial \boldsymbol{r}_{\mathrm{p}}}{\partial u_{\mathrm{p}}} \times \frac{\partial \boldsymbol{r}_{\mathrm{p}}}{\partial \theta_{\mathrm{p}}}\right|}, \\
& \boldsymbol{d}_{\mathrm{p}}=\boldsymbol{n}_{\mathrm{p}} \times \boldsymbol{t}_{\mathrm{p}} .
\end{aligned}
$$

The sign chosen in (38) must provide the direction of $\boldsymbol{n}_{\mathrm{p}}$ towards the surface 'body'.

Equations (35) represent in $S_{\mathrm{p}}$ the generated surface $\Sigma_{\mathrm{g}}$ in three parametric form but with related parameters. Parameter $u_{\mathrm{t}}$ is linear in the equation of meshing when $\Sigma_{\mathrm{t}}$ is a cone, therefore this parameter can be eliminated and the generated surface $\Sigma_{\mathrm{g}}$ can be represented in $S_{\mathrm{p}}$ as

$$
r_{\mathrm{p}}^{(\mathrm{g})}=r_{\mathrm{g}}=r_{\mathrm{g}}\left(\theta_{\mathrm{p}}, \theta_{\mathrm{t}}\right)
$$

We recall that surfaces $\Sigma_{g}$ and $\Sigma_{p}$ have a common line $L_{m}$ where they are in tangency. Surface $\Sigma_{g}$ is 
in tangency with $\Sigma_{\mathrm{t}}$ along the instantaneous line $L_{\mathrm{g}}$ that passes through the current point $M$ of line $L_{\mathrm{m}}$. The tangents to $L_{\mathrm{g}}$ and $L_{\mathrm{m}}$ lie in the plane that passes through $M$ and is tangent to three surfaces $\left(\Sigma_{\mathrm{p}}, \Sigma_{\mathrm{g}}\right.$ and $\Sigma_{\mathrm{t}}$ ) simultaneously.

\section{Optimal approximation of the generated surface $\Sigma_{\mathrm{g}}$ to the ideal surface $\Sigma_{\mathrm{p}}$}

The procedure of optimal approximation of $\Sigma_{\mathrm{g}}$ to $\Sigma_{\mathrm{p}}$ is divided into the following stages: (i) design of grid on $\Sigma_{\mathrm{p}}$, the net of points, where the deviations of $\Sigma_{\mathrm{g}}$ from $\Sigma_{\mathrm{p}}$ will be determined; (ii) determination of the initial function $\beta^{(1)}\left(\theta_{\mathrm{p}}\right)$ for the first iteration; angle $\beta$ determines the orientation of the tool surface $\Sigma_{\mathrm{t}}$ with respect to $\Sigma_{\mathrm{p}}$ (Figs. 1 and 3); (iii) determination of deviations of $\Sigma_{\mathrm{g}}$ from $\Sigma_{\mathrm{p}}$ with the initial function $\beta^{(1)}\left(\theta_{\mathrm{p}}\right)$; (iv) optimal minimization of deviations.

\subsection{Grid on surface $\Sigma_{\mathrm{p}}$}

Figure 5(a) shows the grid on the surface $\Sigma_{\mathrm{p}}$, the net of $(n, m)$ points, where the deviations of $\Sigma_{\mathrm{g}}$ from $\Sigma_{\mathrm{p}}$ are considered. The position vector is $O_{\mathrm{p}} Q_{i, j}=r_{\mathrm{p}}^{(i, j)}$ (Fig. 5(b)). The computation is based on the following procedure:

(i) The desired components $L_{i, j}$ and $R_{i, j}$ of the position vector $r_{\mathrm{p}}^{(i, j)}$ are considered as known.

(ii) Taking into account that

$$
l_{i, j}=z_{\mathrm{p}}^{(i, j)}, \quad R_{i, j}^{2}=\left[x_{\mathrm{p}}^{(i, j)}\left(u_{\mathrm{p}}, \theta_{\mathrm{p}}\right)\right]^{2}+\left[y_{\mathrm{p}}^{(i, j)}\left(u_{\mathrm{p}}, \theta_{\mathrm{p}}\right)\right]^{2},
$$

we will obtain the surface $\Sigma_{\mathrm{p}}$ parameters $\left(u_{\mathrm{p}}^{(i, j)}, \theta_{\mathrm{p}}^{(i, j)}\right)$ for each grid point.

(a)

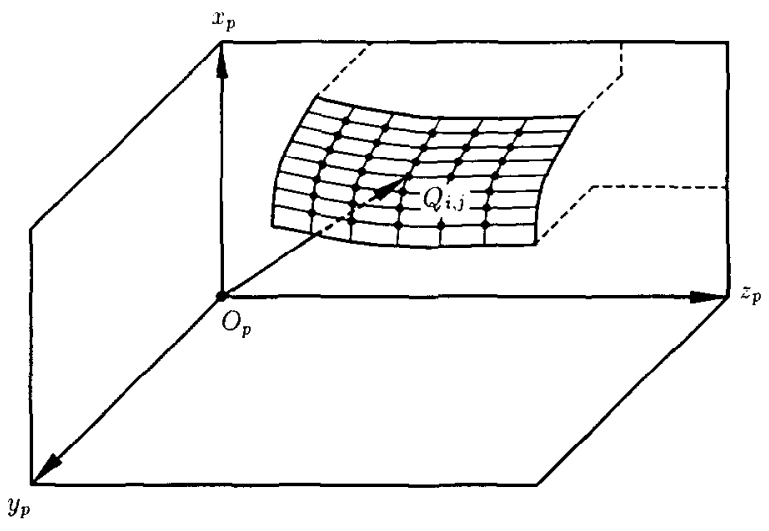

(b)

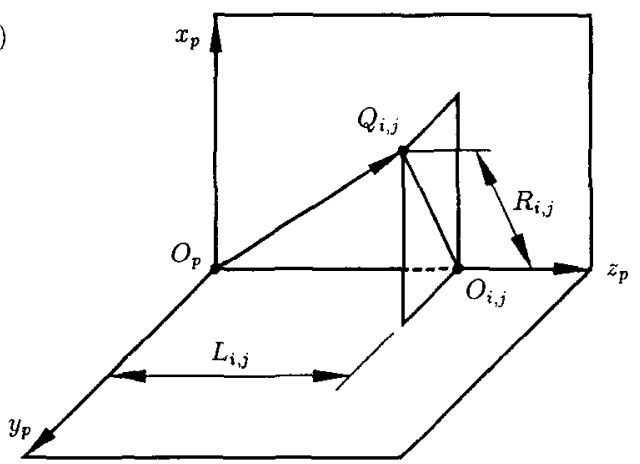

Fig. 5. Grid on surface $\Sigma_{p}$.

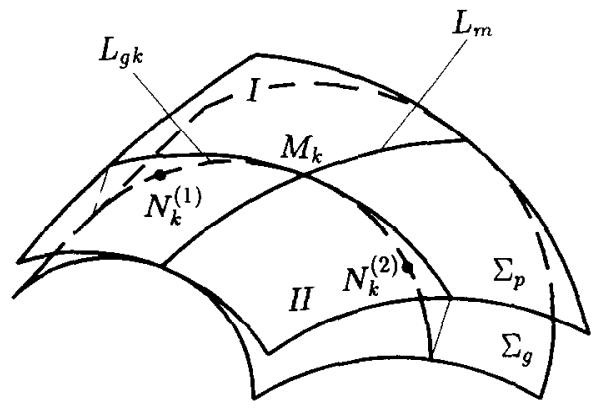

Fig. 6. Determination of maximal deviations along line $L_{g k}$. 


\subsection{Determination of initial function $\beta^{(1)}\left(\theta_{\mathrm{p}}\right)$}

The determination of $\beta^{(1)}\left(\theta_{\mathrm{p}}\right)$ is based on the following idea: the instantaneous direction of $t_{\mathrm{b}}$ (the tool generatrix) with respect to tangent $t_{\mathrm{f}}$ to the mean line $L_{\mathrm{m}}$ (Fig. 3) must provide the minimal value $\left|k_{n}^{(r)}\right|$. Here, $k_{n}^{(r)}$ is the relative normal curvature determined as

$$
k_{\mathrm{n}}^{(\mathrm{r})}=k_{\mathrm{n}}^{(1)}-k_{\mathrm{n}}^{(\mathrm{p})},
$$

where $k_{\mathrm{n}}^{(\mathrm{t})}$ and $k_{\mathrm{n}}^{(\mathrm{p})}$ are the normal curvatures of surfaces $\Sigma_{\mathrm{t}}$ and $\Sigma_{\mathrm{p}}$ along $t_{\mathrm{b}}$. In the casc of a nondevelopable ruled surface $\Sigma_{\mathrm{p}}$, vector $t_{\mathrm{b}}$ can be directed along the asymptote of $\Sigma_{\mathrm{p}}$.

The requirement that $\left|k_{\mathrm{n}}^{(\mathrm{r})}\right|$ is minimal, enables us to determine the function $\beta^{(1)}\left(\theta_{\mathrm{p}}\right)$ numerically. Since we need the derivative $\mathrm{d} \beta / \mathrm{d} \theta_{\mathrm{p}}$, for further computations the function $\beta^{(1)}\left(\theta_{\mathrm{p}}\right)$ is represented analytically as a polynomial function that must satisfy the numerical data obtained for the chosen points of mean line $L_{\mathrm{m}}$.

\subsection{Determination of deviations of $\Sigma_{\mathrm{g}}$ from $\Sigma_{\mathrm{p}}$}

We are able at this stage of the investigation to determine the equation of meshing between surfaces $\Sigma_{\mathrm{t}}$ and $\Sigma_{\mathrm{g}}$, and surface $\Sigma_{\mathrm{g}}$ as discussed in Sections 4 and 5. The computation of deviations of $\Sigma_{\mathrm{g}}$ from $\Sigma_{\mathrm{p}}$ at the grid points is based on the following considerations:

(i) Surfaces $\Sigma_{p}$ and $\Sigma_{g}$ are represented in the same coordinate system $\left(S_{\mathrm{p}}\right)$ by the following vector functions:

$$
r_{\mathrm{p}}\left(u_{\mathrm{p}}, \theta_{\mathrm{p}}\right), \quad r_{\mathrm{g}}\left(\theta_{\mathrm{g}}, \theta_{\mathrm{t}}\right) .
$$

(ii) The position vector $r_{\mathrm{p}}^{(i, j)}$ and surface coordinates $\left(u_{\mathrm{p}}^{(i, j)}, \theta_{\mathrm{p}}^{(i, j)}\right)$ are known for each point $Q_{\mathrm{p}}^{(i, j)}$ of the grid on surface $\Sigma_{p}$.

(iii) Point $Q_{\mathrm{g}}^{(i, j)}$ on surface $\Sigma_{\mathrm{g}}$ corresponds to point $Q_{\mathrm{p}}^{(i, j)}$ on surface $\Sigma_{\mathrm{p}}$. The surface $\Sigma_{\mathrm{g}}$ parameters $\left(\theta_{\mathrm{g}}^{(i, j)}, \theta_{\mathrm{t}}^{(i, j)}\right)$ can be determined by using the following two equations:

$$
y_{\mathrm{g}}^{(i, j)}\left(\theta_{\mathrm{g}}^{(i, j)}, \theta_{\mathrm{t}}^{(i, j)}\right)=y_{\mathrm{p}}\left(u_{\mathrm{p}}^{(i, j)}, \theta_{\mathrm{p}}^{(i, j)}\right), \quad z_{\mathrm{g}}^{(i, j)}\left(\theta_{\mathrm{g}}^{(i, j)}, \theta_{\mathrm{t}}^{(i, j)}\right)=z_{\mathrm{p}}\left(u_{\mathrm{p}}^{(i, j)}, \theta_{\mathrm{p}}^{(i, j)}\right) .
$$

(iv) Due to deviations of $\Sigma_{\mathrm{g}}$ from $\Sigma_{\mathrm{p}}$, we have that $x_{\mathrm{g}}^{(i, j)} \neq x_{\mathrm{p}}^{(i, j)}$. The deviation of $\Sigma_{\mathrm{g}}$ from $\Sigma_{\mathrm{p}}$ at the grid point $Q_{\mathrm{p}}^{(i, i)}$ is determined by the equation

$$
\delta_{i, j}=\boldsymbol{n}_{\mathrm{p}}^{(i, j)} \cdot\left(\boldsymbol{r}_{\mathrm{g}}^{(i, j)}-\boldsymbol{r}_{\mathbf{p}}^{(i, j)}\right)
$$

where $\boldsymbol{n}_{\mathrm{p}}^{(i, j)}$ is the unit normal to surface $\Sigma_{\mathrm{p}}$ at the grid point $Q_{\mathrm{p}}^{(i, j)}$.

The deviation $\delta_{i, j}$ can be positive or negative. We designate as positive such a deviation when $\delta_{i, j}>0$ considering that $\boldsymbol{n}_{\mathrm{p}}^{(i, j)}$ is directed into the 'body' of surface $\Sigma_{\mathrm{p}}$. Positive deviations of $\Sigma_{\mathrm{g}}$ with respect to $\Sigma_{\mathrm{p}}$ provide that $\Sigma_{\mathrm{g}}$ is inside of $\Sigma_{\mathrm{p}}$ and surface $\Sigma_{\mathrm{g}}$ is 'crowned'.

It is not excluded that initially the inequality $\delta_{i, j}>0$ is not yet observed for all points of the grid. Positive deviations $\delta_{i, j}$ can be provided choosing the following options:

(1) choosing a surface of revolution with a circular arc in the axial section instead of a circular cone; a proper radius of the circular arc must be determined.

(2) changing parameter $l_{\mathrm{t}}=\left|\overline{O_{\mathrm{t}} O_{\mathrm{b}}}\right|$ (Figs. 3 and 4); this means that the grinding cone will be displaced along $t_{\mathrm{b}}$ with respect to the mean line $L_{\mathrm{m}}$.

(3) varying the initially chosen function $\beta^{(1)}\left(\theta_{\mathrm{p}}\right)$.

\subsection{Minimization of deviation $\delta_{i, j}$}

Consider that deviations $\delta_{i, j}(i=1, \ldots, n ; j=1, \ldots, m)$ of $\Sigma_{\mathrm{g}}$ with respect to $\Sigma_{\mathrm{p}}$ have been determined at the $(n, m)$ grid points. The minimization of deviations can be obtained by corrections of 
the previously obtained function $\beta^{(1)}\left(\theta_{\mathrm{p}}\right)$. The correction of angle $\beta$ is equivalent to the correction of the angle that is formed by the principal directions on surfaces $\Sigma_{t}$ and $\Sigma_{g}$. The correction of angle $\beta$ can be achieved by turning of the tool about the common normal to surfaces $\Sigma_{t}$ and $\Sigma_{p}$ at their instantaneous point of tangency $M_{\mathrm{k}}$.

The minimization of deviations $\delta_{i, j}$ is based on the following procedure:

Step 1. Consider the characteristic $L_{\mathrm{gk}}$, the line of contact between surfaces $\Sigma_{\mathrm{t}}$ and $\Sigma_{\mathrm{g}}$, that passes through current point $M_{\mathrm{k}}$ of mean line $L_{\mathrm{m}}$ on surface $\Sigma_{\mathrm{p}}$ (Fig. 6). Determine the deviations $\delta_{\mathrm{k}}$ between $\Sigma_{\mathrm{t}}$ and $\Sigma_{\mathrm{p}}$ along line $L_{\mathrm{gk}}$ and find the maximal deviations designated as $\delta_{\max }^{(1)}$ and $\delta_{\mathrm{kmax}}^{(2)}$. Points of $L_{\mathrm{gk}}$ where the deviations are maximal are designated as $N_{\mathrm{k}}^{(1)}$ and $N_{\mathrm{k}}^{(2)}$. These points are determined in regions $I$ and II of surface $\Sigma_{\mathrm{g}}$ with linc $L_{\mathrm{m}}$ as the border. The simultaneous consideration of maximal deviations in both regions enables us to minimize the deviations for the whole surface $\Sigma_{g}$.

Note 1. The deviations of $\Sigma_{\mathrm{t}}$ from $\Sigma_{\mathrm{p}}$ along $L_{\mathrm{gk}}$ are simultaneously the deviations of $\Sigma_{\mathrm{g}}$ from $\Sigma_{\mathrm{p}}$ along $L_{\mathrm{gk}}$ since $L_{\mathrm{gk}}$ is the line of tangency of $\Sigma_{\mathrm{t}}$ and $\Sigma_{\mathrm{g}}$.

Step 2. The minimization of deviations is accomplished by correction of angle $\beta_{\mathrm{k}}$ that is determined at point $M_{\mathrm{k}}$ (Fig. 6). The minimization of deviations is performed locally, for a piece $k$ of surface $\Sigma_{\mathrm{g}}$ with the characteristic $L_{\mathrm{gk}}$. The process of minimization is a computerized iterative process based on the following considerations:

(i) The objective function is represented as

$$
F_{\mathrm{k}}=\min \left(\delta_{\mathrm{kmax}}^{(1)}+\delta_{\mathrm{kmax}}^{(2)}\right) \text {, }
$$

with the constraint $\delta_{i, j} \geqslant 0$.

(ii) The variable of the objective function is $\Delta \beta_{k}$. Then, considering the angle

$\beta_{\mathrm{k}}^{(2)}=\beta_{\mathrm{k}}^{(1)}+\Delta \beta_{\mathrm{k}}$

and using the equation of meshing with $\beta_{\mathrm{k}}$, we can determine the new characteristic, the piece of envelope $\Sigma_{\mathrm{g}}^{(\mathrm{k})}$ and the new deviations. The applied iterations provide the required objective function. The final correction of angle $\beta_{\mathrm{k}}$, we designate as $\beta_{\mathrm{k}}^{\text {(opt) }}$.

Note 2. The new contact line $L_{\mathrm{gk}}^{(2)}$ (determined with $\beta_{k}^{(2)}$ ) differs slightly from the real contact line since the derivative $\mathrm{d} \beta_{k}^{(1)} / \mathrm{d} s$ but not $\mathrm{d} \beta_{\mathrm{k}}^{(2)} / \mathrm{d} s$ is used for determining $L_{\mathrm{gk}}^{(2)}$. However, $L_{\mathrm{gk}}^{(2)}$ is very close to the real contact line.

Step 3. The discussed procedure must be performed for the set of pieces of surfaces $\Sigma_{\mathrm{g}}$ with the characteristic $L_{\mathrm{gk}}$ for each surface piece.

We recall that the deviations for the whole surface must satisfy the inequality $\delta_{i, j} \geqslant 0$. The procedure of optimization is illustrated with a flowchart (Fig. 7).

\section{Curvatures of the ground surface $\Sigma_{\mathrm{g}}$}

The direct determination of curvatures of $\Sigma_{\mathrm{g}}$ by using surface $\Sigma_{\mathrm{g}}$ equations is a complicated problem. The solution to this problem can be substantially simplified using the following approach proposed by the authors: (i) the normal curvatures and surface torsions (geodesic torsions) of surfaces $\Sigma_{p}$ and $\Sigma_{g}$ are equal along line $L_{\mathrm{m}}$, respectively; (ii) the normal curvatures and surface torsions of surfaces $\Sigma_{\mathrm{t}}$ and $\Sigma_{\mathrm{g}}$ are equal along line $L_{\mathrm{g}}$ in terms of curvatures of $\Sigma_{\mathrm{p}}$ and $\Sigma_{\mathrm{t}}$. However, only three of these equations are independent (see below). [3].

The term 'surface torsion' instead of 'geodesic torsion' has been proposed by Nutborne and Martin

Further derivations are based on the following equations: 


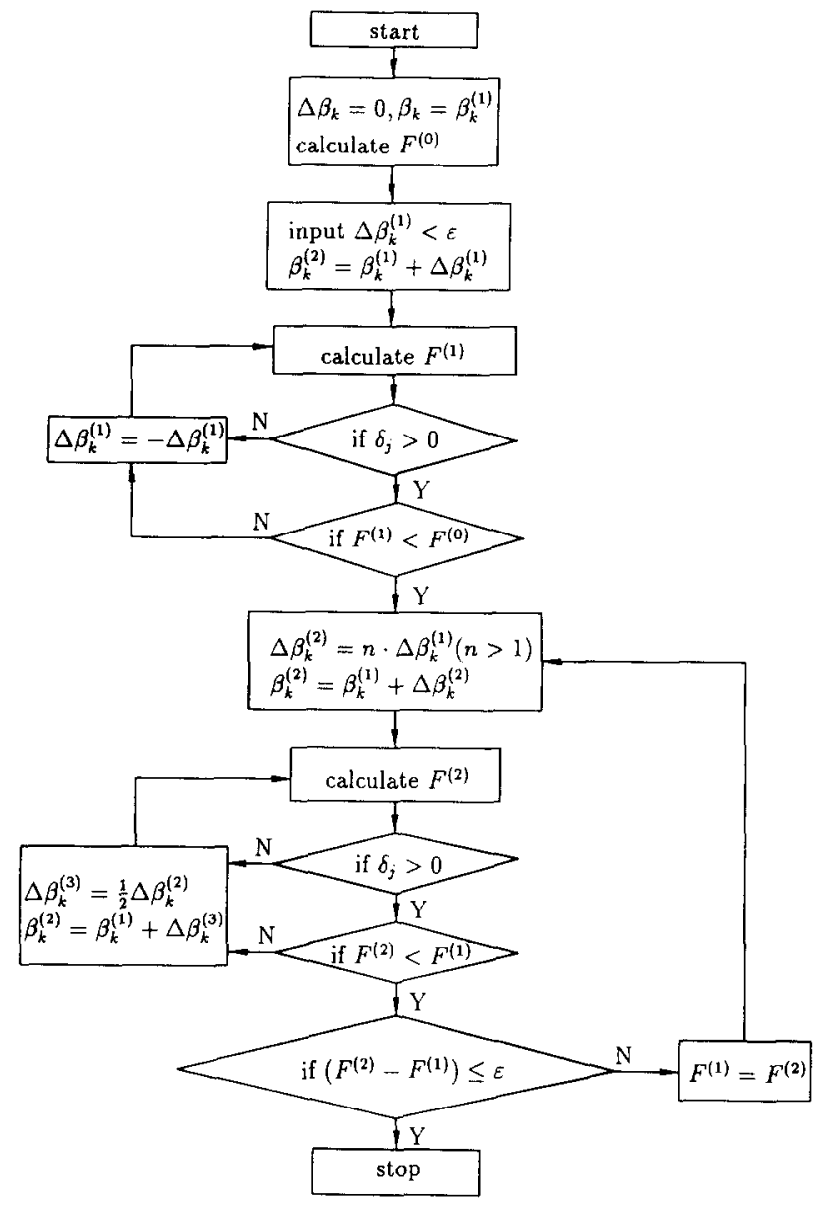

Fig. 7. Flowchart for optimization.

$$
\begin{aligned}
& k_{\mathrm{n}}=k_{\mathrm{I}} \cos ^{2} q+k_{\mathrm{II}} \sin ^{2} q=\frac{1}{2}\left(k_{\mathrm{I}}+k_{\mathrm{II}}\right)+\frac{1}{2}\left(k_{\mathrm{I}}-k_{\mathrm{II}}\right) \cos 2 q, \\
& t=0.5\left(k_{\mathrm{II}}-k_{\mathrm{I}}\right) \sin 2 q,
\end{aligned}
$$

where $k_{\mathrm{I}}$ and $k_{\mathrm{II}}$ are the surface principal curvatures, angle $q$ is formed by unit vectors $\boldsymbol{e}_{\mathrm{i}}$ and $\boldsymbol{e}$ measured counterclockwise from $e_{\mathrm{I}}$ and $e ; e_{\mathrm{I}}$ is the principal direction with principal curvature $k_{\mathrm{I}} ; e$ is the unit vector for the direction where the normal curvature is considered; $t$ is the surface torsion for the direction represented by $e$.

Equation (48) is known as the Euler equation. Equation (49) is known in differential geometry as the Bonnet equation [2] and the Sophia Germain equation [3].

The determination of the principal curvatures and principal directions for $\Sigma_{\mathrm{g}}$ is based on the following computational procedure:

Step 1. Determination of $k_{\mathrm{n}}^{(1)}$ and $t^{(1)}$ for surface $\Sigma_{\mathrm{g}}$ at the direction determined by the tangent to $L_{\mathrm{m}}$. The determination is based on (48) and (49) applied for surface $\Sigma_{\mathrm{p}}$. Recall that $\Sigma_{\mathrm{p}}$ and $\Sigma_{\mathrm{g}}$ have the same values of $k_{\mathrm{n}}$ and $t$ along the abovementioned direction.

Step 2. Determination of $k_{\mathrm{n}}^{(2)}$ and $t^{(2)}$. The designations $k_{\mathrm{n}}^{(2)}$ and $t^{(2)}$ indicate the normal curvature of $\Sigma_{\mathrm{g}}$ and the surface torsion along the tangent to $L_{\mathrm{g}}$. Recall that $k_{\mathrm{n}}^{(2)}$ and $t^{(2)}$ are the same for $\Sigma_{\mathrm{t}}$ and $\Sigma_{\mathrm{g}}$ along $L_{\mathrm{g}}$. We determine $k_{\mathrm{n}}^{(2)}$ and $t^{(2)}$ for surface $\Sigma_{\mathrm{t}}$ using (48) and (49), respectively.

Step 3. We consider at this stage of the computation that for surface $\Sigma_{\mathrm{g}}$ are known $k_{\mathrm{n}}^{(1)}$ and $t^{(1)}, k_{\mathrm{n}}^{(2)}$ and $t^{(2)}$, for two directions with tangents $\tau_{1}$ and $\tau_{2}$ that form the known angle $\mu$ (Fig. 8). Our 


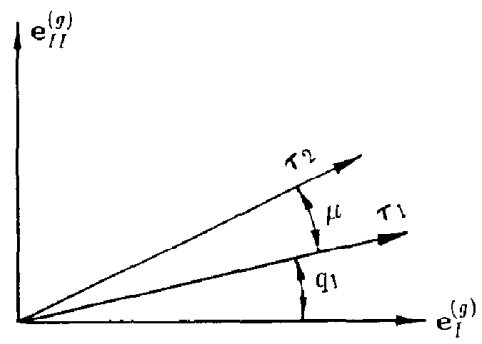

Fig. 8. To determination of principal directions of generated surface $\Sigma_{\mathrm{g}}$.

goal is to determine angle $q_{1}$ (or $q_{2}$ ) for the principal direction $e_{\mathrm{I}}^{(\mathrm{g})}$ and the principal curvatures $k_{\mathrm{I}}^{(\mathrm{g})}$ and $k_{\mathrm{II}}^{(\mathrm{g})}$ (Fig. 8). Using (48) and (49), we can prove that $k_{\mathrm{n}}^{(i)}$ and $t^{(i)}(i=1,2)$ given for two directions represented by $\tau_{1}$ and $\tau_{2}$ are related by

$$
\frac{t_{1}+t_{2}}{k_{\mathrm{n} 2}-k_{\mathrm{n} 1}}=\cot \mu
$$

Step 4. Using (48) and (49), we can derive the following three equations for determination of $q_{i}, k_{\mathrm{I}}^{(\mathrm{g})}$ and $k_{\mathrm{II}}^{(\mathrm{g})}$ :

$$
\begin{aligned}
& \tan 2 q_{1}=\frac{t_{1} \sin 2 \mu}{t_{2}-t_{1} \cos 2 \mu}, \\
& k_{\mathrm{I}}^{(\mathrm{g})}=k_{\mathrm{n}}^{(1)}-t_{1} \tan q_{1}, \\
& k_{\mathrm{II}}^{(\mathrm{g})}=k_{\mathrm{n}}^{(1)}+t_{1} \cot q_{1} .
\end{aligned}
$$

Equation (51) provides two solutions for $q_{1}\left(q_{1}^{(2)}=q_{1}^{(1)}+90^{\circ}\right)$ and both are correct. We choose the solution with the smaller value of $q_{1}$.

\section{Execution of motions on computer numerically controlled (CNC) machine: the 'Phoenix' machine}

The process discussed above for generation of $\Sigma_{\mathrm{g}}$ can be accomplished on a multi-degree-of-freedom $\mathrm{CNC}$ machine. In the following discussions, we consider as an example of the $\mathrm{CNC}$ machine, the 'Phoenix' machine, designed by the Gleason Works (Fig. 9). This machine is provided with six degrees of freedom for three rotational motions, and three translational motions. The translational motions are performed in three mutually perpendicular directions. Two of the rotational motions are provided as the rotation of the workpiece with surface $\Sigma_{\mathrm{g}}$ and the rotation that enables us to change the angle between the axes of the workpiece with the to-be generated surface $\Sigma_{\mathrm{g}}$ and the tool with surface $\Sigma_{\mathrm{t}}$. The sixth rotational motion is provided as the rotation of the tool about its axis, and generally is not related to the process for generation.

The 'workpiece' is the piece of metal that must be provided with the desired surface $\Sigma_{\mathrm{g}}$.

\subsection{Coordinate systems applied for $C N C$}

Coordinate systems $S_{\mathrm{t}}\left(x_{\mathrm{t}}, y_{\mathrm{t}}, z_{\mathrm{t}}\right)$ and $S_{\mathrm{p}}\left(x_{\mathrm{p}}, y_{\mathrm{p}}, z_{\mathrm{p}}\right)$ are rigidly connected to the tool and the workpiece, respectively (Fig. 10). Coordinate system $S_{\mathrm{m}}$ performs translational motion along axis $z_{\mathrm{t}}$ with respect to the frame of 'Phoenix'.

Coordinate system $S_{\mathrm{h}}$ performs translational motions with respect to $S_{\mathrm{m}}$. Coordinate system $S_{\mathrm{t}}$ performs rotational motion with respect to $S_{\mathrm{h}}$ about the $z_{\mathrm{h}}$-axis. Coordinate system $S_{\mathrm{e}}$ performs rotational motion with respect to $S_{\mathrm{m}}$ about the $y_{\mathrm{m}}$-axis. Axes of the coordinate system $S_{\mathrm{d}}$ are parallel to 


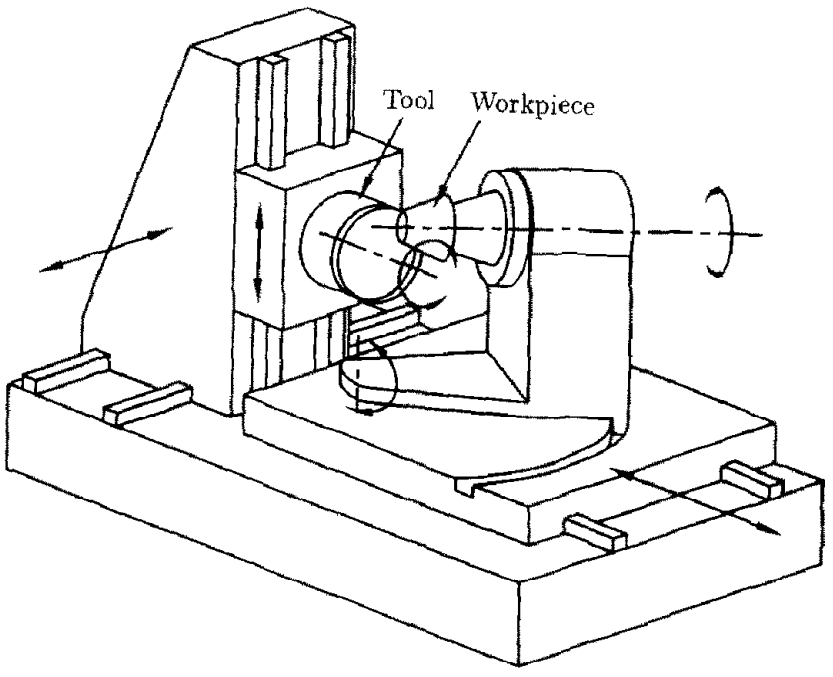

Fig. 9. Schematic of 'Phoenix' machine.

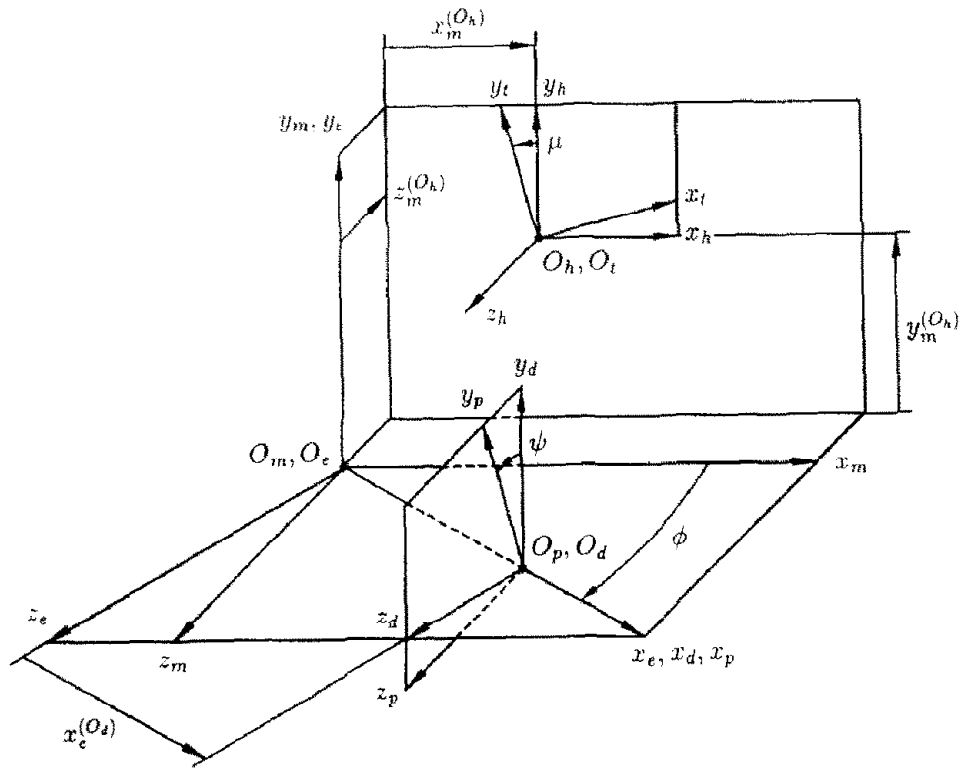

Fig. 10. Coordinate systems used for 'Phoenix' machine.

the respective axes of $S_{e}$; the location of origin $O_{\mathrm{d}}$ with respect to $O_{\mathrm{e}}$ is determined with the parameter $x_{\mathrm{e}}^{\left(O_{\mathrm{d}}\right)}=$ const. Coordinate system $S_{\mathrm{p}}$ performs rotational motion with respect to $S_{\mathrm{d}}$ about the $x_{\mathrm{d}}$-axis.

\subsection{Execution of motions}

Execution of motions of the 'Phoenix' machine for the generation of conventional spiral bevel gears and hypoid gears has bcen discussed by Goldrich [5]. The execution of motions for the method for generation proposed in this paper is based on the following matrix equations (Figs. 2, 3, 4, 10):

$$
\begin{aligned}
& \boldsymbol{L}_{\mathrm{pt}}^{(\mathrm{C})}(\mu, \psi, \phi)=\boldsymbol{L}_{\mathrm{pt}}^{(\mathrm{G})}\left(\theta_{\mathrm{p}}\right), \\
& \boldsymbol{M}_{\mathrm{pt}}^{(\mathrm{C})}\left(\mu, \psi, \phi, x_{\mathrm{m}}^{\left(O_{\mathrm{h}}\right)}, y_{\mathrm{m}}^{\left(O_{\mathrm{h}}\right)}, z_{\mathrm{m}}^{\left(O_{\mathrm{h}}\right)}\right)\left[\begin{array}{llll}
0 & 0 & 0 & 1
\end{array}\right]^{\mathrm{t}}=\boldsymbol{M}_{\mathrm{pt}}^{(\mathrm{G})}\left(\theta_{\mathrm{p}}\right)\left[\begin{array}{llll}
0 & 0 & 0 & 1
\end{array}\right]^{\mathrm{t}},
\end{aligned}
$$

where

$$
L_{\mathrm{pt}}^{(\mathrm{C})}=L_{\mathrm{pd}}(\psi) L_{\mathrm{de}} L_{\mathrm{em}}(\phi) L_{\mathrm{rhh}} L_{\mathrm{ht}}(\mu)
$$


and $\boldsymbol{L}_{\mathrm{de}}$ and $\boldsymbol{L}_{\mathrm{mh}}$ are unitary matrices.

$$
L_{\mathrm{pt}}^{(\mathrm{G})}=L_{\mathrm{pf}}\left(\theta_{\mathrm{p}}\right) L_{\mathrm{fb}}\left(\beta\left(\theta_{\mathrm{p}}\right)\right) L_{\mathrm{bt}}\left(\theta_{\mathrm{t}}^{*}\right)
$$

The superscript $\mathrm{C}$ indicates that the coordinate transformation is performed for the CNC machine. The superscript $G$ indicates the coordinate transformation when the generation of $\Sigma_{g}$ by the method proposed in this paper is considered. Parameter $\boldsymbol{\theta}_{\mathrm{t}}^{*}$ is constant and designates the chosen generatrix of the tool surface with the unit vector $t_{\mathrm{b}}$ (Figs. 2 and 4 ).

Using matrix equation (54), we obtain the functions $\psi\left(\theta_{\mathrm{p}}\right)$ and $\phi\left(\theta_{\mathrm{p}}\right)$ that are required for execution of rotational motions. Angle $\mu$ represents the rotation angle of the tool and it can be chosen deliberately since the tool surface $\Sigma_{\mathrm{t}}$ is a surface of revolution.

Matrix equation (55) provides that the position vector $\overline{O_{\mathrm{p}} O_{\mathrm{t}}}$ will be the same for both cases of coordinate transformation. Using this equation, we can determine the functions $x_{\mathrm{m}}^{\left(O_{\mathrm{h}}\right)}\left(\theta_{\mathrm{p}}\right), y_{\mathrm{m}}^{\left(O_{\mathrm{h}}\right)}\left(\theta_{\mathrm{p}}\right)$ and $z_{\mathrm{m}}^{\left(O_{\mathrm{h}}\right)}\left(\theta_{\mathrm{p}}\right)$ for the execution of translational motions.

\section{Numerical example: grinding of Archimedes' worm surface}

The worm surface shown in Fig. 11 is a ruled undeveloped surface formed by the screw motion of the straight line $\overline{K N}\left(|\overline{K N}|=u_{\mathrm{p}}\right)$. The screw motion is performed in coordinate system $S_{\mathrm{p}}$ (Fig. 11(b)). The to-be ground surface $\Sigma_{\mathrm{p}}$ is represented in $S_{\mathrm{p}}$ as

$$
r_{\mathrm{p}}=u_{\mathrm{p}} \cos \alpha \cos \theta_{\mathrm{p}} i_{\mathrm{p}}+u_{\mathrm{p}} \cos \alpha \sin \theta_{\mathrm{p}} j_{\mathrm{p}}+\left(p \theta_{\mathrm{p}}-u_{\mathrm{p}} \sin \alpha\right) k_{\mathrm{p}}
$$

where $u_{\mathrm{p}}$ and $\theta_{\mathrm{p}}$ are the surface parameters.

The surface unit normal is

$$
n_{\mathrm{p}}=\frac{N_{\mathrm{p}}}{\left|N_{\mathrm{p}}\right|}, \quad N_{\mathrm{p}}=\frac{\partial r_{\mathrm{p}}}{\partial u_{\mathrm{p}}} \times \frac{\partial r_{\mathrm{p}}}{\partial \theta_{\mathrm{p}}}
$$

Thus,

(a)

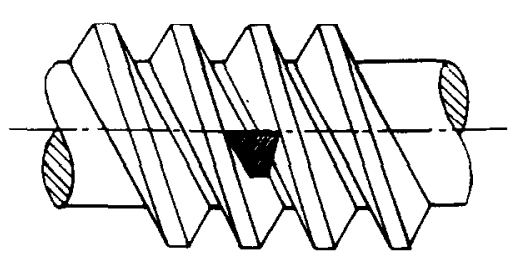

(b)

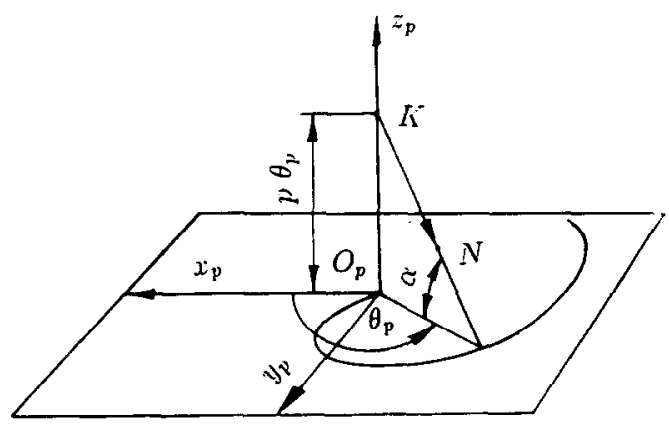

Fig. 11. Surface of Archimedes' worm. 


$$
\boldsymbol{n}_{\mathrm{p}}=\frac{1}{\left(u_{\mathrm{p}}^{2}+p^{2}\right)^{0.5}}\left[\begin{array}{c}
p \sin \theta_{\mathrm{p}}+u_{\mathrm{p}} \sin \alpha \cos \theta_{\mathrm{p}} \\
-p \cos \theta_{\mathrm{p}}+u_{\mathrm{p}} \sin \alpha \sin \theta_{\mathrm{p}} \\
u_{\mathrm{p}} \cos \alpha
\end{array}\right] \quad(\text { provided } \cos \alpha \neq 0) .
$$

The design data are: number of threads $N_{1}=2$; axial diametral pitch $P_{\text {ax }}=8$ in $^{-1} ; \alpha=20^{\circ}$; the radius of the pitch cylinder is $\mathbf{1 . 1 2 5}$ in. The remaining design parameters are determined from the following equations:

(i) The screw parameter is

$$
p=\frac{N_{1}}{2 P_{\mathrm{ax}}}=0.125 \text { in }
$$

(ii) The lead angle is

$$
\tan \lambda_{\mathrm{p}}=\frac{p}{r_{\mathrm{p}}}=\frac{0.25}{1.125}, \quad \lambda_{\mathrm{p}}=12.5288^{\circ}
$$

The mean line is determined as

$$
r_{\mathrm{p}}\left(u_{\mathrm{m}}, \theta_{\mathrm{p}}\right), \quad u_{m}=\frac{\left(r_{\mathrm{p}}+\frac{1}{P_{\mathrm{ax}}}\right)+\left(\mathrm{r}_{\mathrm{p}}-\frac{1.25}{P_{\mathrm{ax}}}\right)}{2 \cos \alpha}=\frac{r_{\mathrm{p}}-\frac{0.5}{P_{\mathrm{ax}}}}{\cos \alpha}=1.1263 \mathrm{in} .
$$

where $1 / P_{\mathrm{ax}}$ and $1.25 / P_{\mathrm{ax}}$ determine the addendum and dedendum of the worm.

The worm is ground by a cone with the apex angle $\gamma_{\mathrm{t}}=30^{\circ}$, and an outside diameter of 8 in.

The initial angle $\beta^{(1)}=-88.0121^{\circ}$ provides the coincidence of both generatrices of the cone and the Archimedes' worm. The maximal deviation of the ground surface $\Sigma_{\mathrm{g}}$ from the ideal surface $\Sigma_{\mathrm{p}}$ with the above value of $\beta^{(1)}$ is $3 \mu \mathrm{m}$.

The optimal angle $\beta^{(\mathrm{opt})}=-94.6788^{\circ}$ has been determined by the developed optimization method. The deviations of the ground surface $\Sigma_{\mathrm{g}}$ from $\Sigma_{\mathrm{p}}$ with the optimal $\beta^{(\mathrm{opt})}$ are positive and the maximal deviation has been reduced to $0.35 \mu \mathrm{m}$ (Fig. 12).

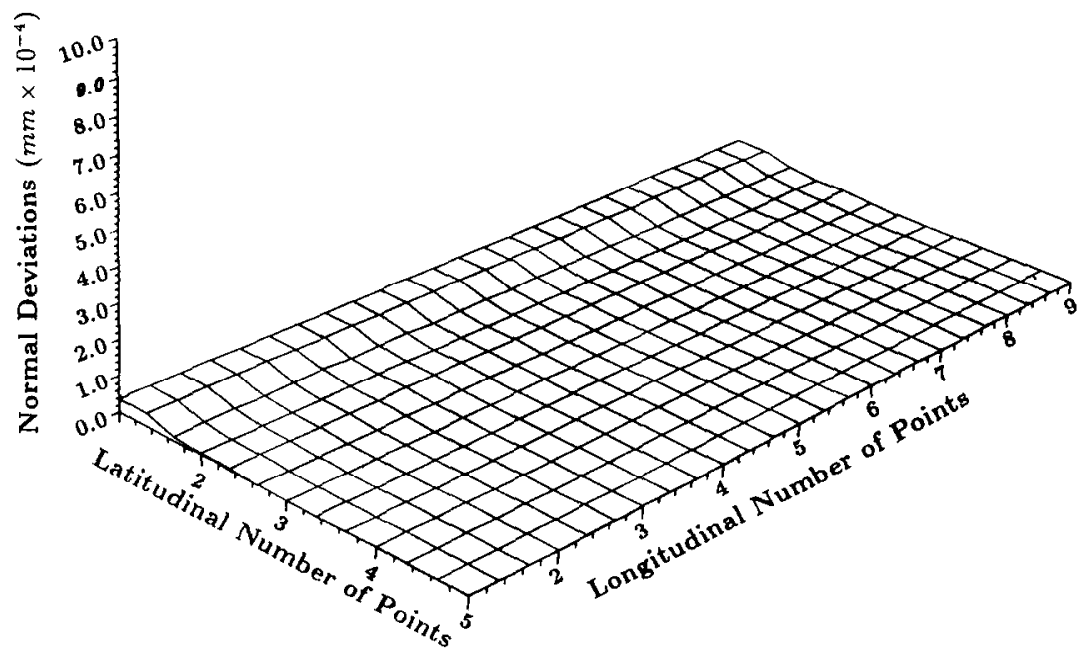

Fig. 12. Deviations of the ground surface $\Sigma_{\mathrm{g}}$ from ideal surface $\Sigma_{\mathrm{p}}$ of Archimedes' worm. 


\section{Conclusion}

(1) A computerized method for generation (by grinding or cutting) of a surface $\Sigma_{g}$ with optimal approximation to the ideal surface $\Sigma_{\mathrm{p}}$ has been developed. The tool used for generation is provided by a surface of a circular cone or a surface of revolution. The required motions of the tool with respect to the to-be-generated surface are executed on a computerized multi-degree-of-freedom machine.

(2) The theory of the proposed method for generation, the algorithm for execution of motions in the process for generation, and the procedure for optimal approximation of $\Sigma_{\mathrm{g}}$ to $\Sigma_{\mathrm{p}}$ have been developed.

(3) An effective approach for the determination of curvatures of the generated surface $\Sigma_{\mathrm{g}}$ has been developed.

(4) A numerical example of generation of an Archimedes' worm has been presented.

\section{Acknowledgment}

This work has received financial support from the NASA Lewis Research Center and the Gleason Memorial Fund.

\section{References}

[1] F.L. Litvin, Theory of Gearing, NASA Reference Publication 1212 (NASA, 1989).

[2] J. Favard, Cours de Geometrie Differentielle Localle (Gauthiers-Villars, Paris, 1957).

[3] A. Nutbourne and R. Matrin, Differential Geometry Applied to Curve and Surface Design, Vol. 1: Foundation (Wiley, New York, 1988).

[4] Н. Goldstein, Classical Mechanics (Addison-Wesley, Reading, MA, 1980).

[5] R.N. Goldrich, Theory of 6-axis CNC generation of spiral bevel and hypoid gears, AGMA Technical Paper 89FTM9, 1989. 